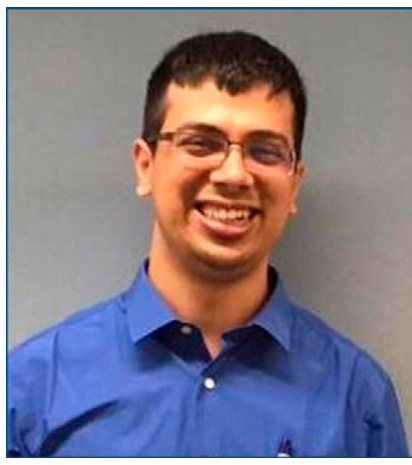

BISHAL GYAWALI, MD, PhD

Bishal Gyawali, MD, PhD, is a medical oncologist with work experience in various low- and high-income countries including Nepal, Japan, United States, and Canada. Currently, he is an Assistant Professor in the Department of Public Health Sciences, Scientist in the Division of Cancer Care and Epidemiology, and a Clinical Fellow in the Department of Medical Oncology at Queen's University, Canada, and an affiliated faculty at the Program On Regulation, Therapeutics and Law at Brigham and Women's Hospital.

Dr. Gyawali also serves as a medical consultant for the not-for-profit Anticancer Fund in Belgium, and is on Editorial and Advisory Boards for multiple medicine and oncology journals. His areas of interests include cancer policy, global oncology, evidence-based oncology, financial toxicities of cancer treatment, clinical trial methods, and supportive care. He is an advocate of "cancer groundshot," a term he coined to imply that investments should be made on proven high-value interventions in cancer care that are easy to implement globally and are affordable. Dr. Gyawali tweets at @oncology_bg.

doi: $10.6004 /$ jnccn.2019.7317

The ideas and viewpoints expressed in this commentary are those of the author and do not necessarily represent any policy, position, or program of NCCN.

\section{Multiple Approvals, Celestial Prices, Unimproved Outcomes: The Tale of Cost-Ineffective Drugs in Hepatocellular Carcinoma}

\author{
Bishal Gyawali, MD, PhD
}

H epatocellular carcinoma ( $\mathrm{HCC}$ ) is one of the most lethal cancers. In the United States, the 5 -year survival rate is only $18 \%$, and rates for advanced or metastatic disease are much poorer, at around $2 \% .{ }^{1}$ In 2007 , sorafenib became the first targeted therapy to show an improvement in overall survival (OS) for patients with advanced $\mathrm{HCC}$ in a randomized controlled trial (RCT). Although the gains in median OS were modest (10.7 vs 7.9 months), this was heralded as a big victory for a disease with no therapeutic options at all. ${ }^{2}$

A decade of silence then followed, until 2017, when regorafenib became the second drug (the first drug in second line) to be approved by the FDA for treatment of advanced HCC. However, this approval heralded an "open floodgate" of drug approvals in HCC. Today, 7 novel noncytotoxic drugs for HCC have been FDAapproved and are listed in the NCCN Clinical Practice Guidelines in Oncology (NCCN Guidelines) for Hepatobiliary Cancers ${ }^{3}$ (Table 1).

Lenvatinib is the only other approved first-line therapy and the only therapy to be compared against an active control (sorafenib). However, it was compared against sorafenib in a noninferiority design trial, although lenvatinib is not cheaper, easier to administer, or less toxic than sorafenib. ${ }^{4}$

Regorafenib became the first drug to receive approval for use in patients whose disease has progressed on first-line sorafenib, after it showed improved OS versus placebo in a phase III RCT. However, the patients selected for this trial were fit and had good liver function, whereas those with HCC-especially those whose disease had already progressed on first-line sorafenib-usually have poor liver function. ${ }^{5}$

Then 2 immunotherapies, nivolumab and pembrolizumab, were approved for second-line treatment. When increased mortality with PD-1 inhibitors in patients with multiple myeloma was discovered in RCTs, leading to these trials being halted, the FDA cautioned the oncology community on the need for RCTs rather than relying on single-arm trials for assessing true efficacy and safety of any drug. ${ }^{6}$ Thus, it is a little surprising that the FDA approved nivolumab and pembrolizumab for $\mathrm{HCC}$ in secondline treatment based only on durable responses seen in single-arm trials. However in a phase III trial, pembrolizumab failed to meet the co-primary end points of both OS and PFS, and the FDA has not yet made any decision about this indication. Although the drug remains in the NCCN Guidelines, the recommendation was changed from category $2 \mathrm{~A}$ to a category $2 \mathrm{~B}$ designation.

Ramucirumab was tested in the second-line setting for advanced HCC, where it showed improved OS versus placebo in the subgroup of patients with an $\alpha$-fetoprotein level $\geq 400 \mathrm{ng} / \mathrm{mL}$. The FDA approved ramucirumab for this indication on May 10 2019, and it is listed in the NCCN Guidelines ${ }^{3}$ as a category 1 recommendation.

In January 2019, cabozantinib received FDA approval for treatment of patients with $\mathrm{HCC}$ that has progressed on first-line sorafenib (also category 1 in the NCCN

See page 669 for related article. 
Guidelines ${ }^{3}$ ). This approval was based on improved OS versus placebo in the CELESTIAL trial (ClinicalTrials.gov identifier: NCT01908426).

Having 7 drugs today sounds better than having 1 drug or none a decade ago, but the billion dollar question is whether outcomes for patients with advanced liver cancer have improved as a result. Some of the statistics are concerning. Although it is too early to observe the effect of new drug approvals, no improvement in survival has been seen for patients with advanced HCC over the past 3 decades. ${ }^{1}$ However, even the effect of sorafenib, the first drug approved for HCC, seems to be nearly negligible. Deaths due to liver cancer actually increased between 2011 and 2015. ${ }^{1}$ A study using the SEER database confirmed that the efficacy of sorafenib seen in the pivotal RCT did not replicate to real-world effectiveness: the median OS for patients receiving placebo in the RCT was higher than the OS for patients receiving sorafenib in the SEER database. ${ }^{7}$ This suggests that the patient selection criteria in the trial does not represent most "realworld" patients who will receive the drug once it is approved. The trials for the newer drugs approved for HCC include similarly highly selected patients.

Furthermore, Table 1 shows surprising findings. First, all of the second-line drugs improve median OS by $<3$ months (range, 1.2-2.8 months) versus placebo. Second, the median OS of the control arm in these trials is between 7 and 8 months, which is surprisingly high for patients who have already experienced disease progression on first-line therapy, given that the median survival in patients with newly diagnosed advanced HCC is usually 8 to 10 months. Indeed, the previously mentioned real-world study showed a median OS of 5 months for patients treated with first-line sorafenib. To have a median OS of 8 months in the placebo cohort reflects extraordinary patient selection that is not representative of the average patient with $\mathrm{HCC}$ seen in clinic.

Despite these doubts about efficacy, and the risk of severe, serious, and even fatal adverse events with these drugs, they come at a substantial price, with monthly costs of treatment ranging from $\$ 13,000$ to $\$ 22,000$. Are the benefits from these drugs worth the price? Cost-effectiveness studies help answer this question.

Soto-Perez-de-Celis et $\mathrm{al}^{8}$ publish such a cost-effective analysis elsewhere in this issue in which they use data from the CELESTIAL trial. They found that cabozantinib was not costeffective, which is not surprising. What is surprising is that the incremental cost-effectiveness ratio (ICER) for cabozantinib was $>\$ 1$ million and that when the upper limit of the 95\% confidence interval of the hazard ratio for OS was used in a sensitivity analysis, placebo became more cost-effective than cabozantinib. The authors concluded that there were no scenarios $(10 \%, 20 \%$, or $30 \%$ discounts on the price of cabozantinib) in which the drug could be considered costeffective; the ICER always remained $>\$ 200,000$. At a willingness-to-pay threshold of $\$ 100,000$ per quality-adjusted life year (QALY), the cost of cabozantinib would have to be $\$ 50$ per pill to be cost-effective; currently it is approximately $\$ 734$ per pill.

Soto-Perez-de-Celis et $\left.\right|^{8}{ }^{8}$ provide food for thought. Even if the price of cabozantinib is reduced by $93 \%$, it would still be considered not cost-effective despite a willingness to pay up to $\$ 100,000$ for a gain in QALY. Furthermore, these numbers assume that the OS seen in the CELESTIAL trial will hold true for less carefully selected patients, which, as noted previously, may be too optimistic. Can we, as a society, afford to continue paying such extraordinary prices for drugs that in an ideal scenario (among the fittest patients) improve OS by perhaps 2 months?

Patients with cancer need more treatment options, but they need better treatments, not just any treatment. Multiple drug options are meaningless if none improve outcomes nor are cost-effective. Would we recommend that patients without insurance pay for these drugs out of pocket? If not, is it ethical for us to ask that public money in the form of Medicare, Medicaid, or other public health insurance be spent on them?

We need to take a stance. We can no longer ignore costeffectiveness in our policy and even clinical decisions. The

Table 1. Drugs Approved for Treatment of Advanced Hepatocellular Cancer

\begin{tabular}{|c|c|c|c|c|c|c|c|}
\hline Drug & Control Arm & Line & \multicolumn{2}{|c|}{ Median OS, mo } & $\Delta$ OS & HR $(95 \% \mathrm{Cl})$ & PMID \\
\hline Sorafenib & Placebo & First & 10.7 & 7.9 & 2.8 & $0.69(0.55-0.87)$ & 18650514 \\
\hline Lenvatinib & Sorafenib & First & 13.6 & 12.3 & 1.3 & $0.92(0.79-1.06)$ & 29433850 \\
\hline Regorafenib & Placebo & Second & 10.6 & 7.8 & 2.8 & $0.63(0.50-0.79)$ & 27932229 \\
\hline Nivolumab & NA & Second & NA & NA & NA & NA & 28434648 \\
\hline Ramucirumab & Placebo & $\begin{array}{l}\text { Second } \\
\text { (AFP } \geq 400 \mathrm{ng} / \mathrm{mL} \text { ) }\end{array}$ & 8.5 & 7.3 & 1.2 & $0.71(0.53-0.95)$ & 30665869 \\
\hline Cabozantinib & Placebo & Second & 10.2 & 8.0 & 2.2 & $0.76(0.63-0.92)$ & 29972759 \\
\hline
\end{tabular}

Abbreviations: AFP, $\alpha$-fetoprotein; HR, hazard ratio; NA, not available; OS, overall survival; PMID, PubMed manuscript identifier for the trial. 
FDA cannot consider cost-effectiveness in its decisions, but we, the oncology community (oncology healthcare professionals, societies, guideline committees) can and must, especially in situations where the benefits are uncertain and/or marginal. A common feature among all drugs for HCC is that HCC was not their first indication; that is, these drugs were already approved for other indications. Thus, they were already in the market and their prices were known. For trials in which a drug already in market is being tried for a new indication, we should demand that cost-effectiveness analysis by an independent group be published simultaneously. Indeed, if we agree that drug toxicities must be reported with trial publications, so should data on financial toxicity.

For example, a cost-effectiveness analysis for necitumumab in metastatic squamous cell non-small cell lung cancer (NSCLC) was performed even before the drug received FDA approval. The analysis concluded that it should be priced at $\$ 563$ to $\$ 1,309$ per cycle for it to be considered costeffective with a willingness-to-pay threshold of $\$ 100,000$ to
$\$ 200,000$ per QALY. ${ }^{9}$ However, when the drug was approved, it was launched at a price of more than $\$ 10,000$ per cycle. In the 2017 update of the NCCN Guidelines for NSCLC, ${ }^{10}$ the panel took a commendable and rare step of deleting the necitumumab/cisplatin/gemcitabine regimen from its guidelines based on poor cost-effectiveness. It was an unprecedented action taken by NCCN regarding a regimen that improved OS in an RCT and received FDA approval.

Because the FDA doesn't take cost-effectiveness into account when making approval decisions, cost-effectiveness studies usually end up being merely intellectual exercises-one may not need a formal analysis to infer that these prices are out of proportion to their benefits. Sometimes, however, cost-effectiveness studies reveal prices that are so dramatically high and disproportionate to benefits that it can help wake up the oncology community and encourage us to reconsider policy. Therein lies the importance of costeffectiveness analyses: they help us uncover other necitumumabs in oncology.

\section{References}

1. Siegel RL, Miller KD, Jemal A. Cancer statistics, 2019. CA Cancer J Clin 2019;69:7-34

2. Llovet JM, Ricci S, Mazzaferro V, et al. SHARP Investigators Study Group. Sorafenib in advanced hepatocellular carcinoma. N Engl J Med 2008;359: 378-390.

3. Benson AB III, D'Angelica MI, Abbott DE, et al. NCCN Clinical Practice Guidelines in Oncology: Hepatobiliary Cancers. Version 2.2019. Accessed May 9, 2019. To view the most recent version, visit NCCN.org.

4. Gyawali B, Kesselheim AS. US Food and Drug Administration approval of new drugs based on noninferiority trials in oncology: a dangerous precedent? JAMA Oncol 2019;5:607-608.

5. Gyawali B, Prasad V. Health policy: me-too drugs with limited benefits: the tale of regorafenib for HCC. Nat Rev Clin Oncol 2017;14:653-654.

6. Gormley NJ, Pazdur R. Immunotherapy combinations in multiple myeloma-known unknowns. N Engl J Med 2018;379:1791-1795.

7. Sanoff HK, Chang Y, Lund JL, et al. Sorafenib effectiveness in advanced hepatocellular carcinoma. Oncologist 2016;21:1113-1120.

8. Soto-Perez-de-Celis E, Aguiar PN Jr, Cordón M, et al. Cost-effectiveness of cabozantinib in the second-line treatment of advanced hepatocellular carcinoma. J Natl Compr Canc Netw 2019;17:669-675.

9. Goldstein DA, Chen $\mathrm{Q}$, Ayer T, et al. Necitumumab in metastatic squamous cell lung cancer: establishing a value-based cost. JAMA Oncol 2015;1:1293-1300.

10. Ettinger DS, Wood DE, Aggarwal C, et al. NCCN Clinical Practice Guidelines in Oncology: Non-Small Cell Lung Cancer. Version 4.2019. Accessed May 9, 2019. To view the most recent version, visit NCCN.org. 